\section{History is (also) telling stories!}

\author{
Ein Interview von Rosemarie Grantyn
}

Der aktuelle Präsident der International Society for the History of Neurosciences und langjährige Direktor des Max-Planck-Instituts für Psychiatrie in München-Martinsried, Professor Georg Kreutzberg erinnert sich...

Neuroforum: Ihr Ururgroßvater Georg Kreutzberg hat im Rheinland Wein angebaut, dabei den Apollinaris-Brunnen entdeckt und ist auf die Heilquellen gestoßen, welche aus Ihrem Geburtsort den Kurort Bad Neuenahr machten. Großvater und Vater waren dort Chefärzte eines Stiftungskrankenhauses. Als Kind sind Sie aber der Faszination des Rheingesteins verfallen. Was hat Sie dann dazu gebracht, sich den Neurowissenschaften zu verschreiben?

Georg Kreutzberg: Die „-wissenschaften“ sind wohl meiner grundsätzlichen Neugier geschuldet! Dass es „Neuro“ und nicht „Geo“ wurde, hing mit dem Wunsch zusammen, Arzt zu werden. Von 1951 bis 1957 habe ich in Freiburg Medizin studiert. Dabei näherte ich mich den Neurowissenschaften über die Chemie, dann Biochemie und habe mit einer Untersuchung zum Tryptophan-Stoffwechsel bei psychiatrischen Erkrankungen promoviert.

Neuroforum: Das fiel ja in die Zeit, als so bedeutende Neurophysiologen wie Rolf Hassler, Paul A. Hoffmann und Richard Jung in Freiburg wirkten. Sie aber haben sich zunächst gegen die neurophysiologische Forschung für die Psychiatrie entschieden. Was hat Sie angelockt?

Georg Kreutzberg: Die Psychiatrie hatte damals eine starke philosophische Komponente und verstand psychische Erkrankungen entweder aus der Perspektive der Freud'schen Psychoanalyse oder der Binswanger'schen Daseinsanalyse, bediente sich also existenzphilosophischer Denkmodelle. Sartre und Merlot-Ponty waren für uns Pflichtlektüre, aber ganz besonders einflussreich in Freiburg war Heidegger, dessen Ausstrahlung stets für überfüllte Hörsäle sorgte. So kam es, dass sich die Psychiatrie - und dies ist gewiss der besonderen wissenschaftlich-historischen Situation der 50iger Jahre in Deutschland geschuldet - zum Teil der Sprache der Philosophie bediente. Wir „heideggerten“ alle, sprachen zum Beispiel bei der Hypochondrie von einer gestörten Wahrnehmung der „Leiblichkeit". Leib und Geist waren damals nicht unbedingt eine Einheit, das Denken, Fühlen nicht unbedingt ein ,emergent property of the brain"...

Neuroforum: Mit solcherart theoretischer Betrachtung normaler und gestörter Gehirnfunktionen ließen Sie es aber nicht bewenden, Sie wollten experimentell arbeiten. Wo fanden Sie zu jener Zeit, in dem wirtschaftlich schon etwas erstarkten, aber wissenschaftlich noch keineswegs erholten Nachkriegsdeutschland Deutschland ihre Lehrer?

Georg Kreutzberg: Die fünfziger Jahre waren zweifellos eine sehr dynamische Zeit, auch eine Zeit der Wiederentdeckung intellektueller und kultureller Werte. Alles, was die Nazis in Kunst, Literatur und Philosophie verboten hatten, wurde dieser ersten Nachkriegsgeneration explosionsartig zuteil. Aber in der Wissenschaft fehlten erfahrene Lehrer und Vorbilder! Um diese zu finden, gingen viele ins Ausland, besonders in die USA. Technologisch gab es einen gewaltigen Abstand zu den Ländern im angelsächsischen Raum, wo die Methoden der Zellbiologie bereits Einzug in die Neuroanatomie und Neurophysiologie gehalten hatten. Deshalb war ich auch sehr dankbar für die Gelegenheit, ein Jahr in Boston zu verbringen, und zwar am Department of Psychology, am Massachusetts Institute of Technology.

Neuroforum: Das war in den Jahren 1964-65. Haben Sie von dem Boom der Wissenschaften im Gefolge des „Sputnik-Schocks“ und der anschließenden großzügige Forschungsfinanzierung während der Kennedy-Ära in den USA profitieren können?

Georg Kreutzberg: Natürlich! Am MIT war dies besonders zu spüren! Dort wurde 1964 von dem eminenten kognitiven Psychologen Hans-Lukas (Luke) Teuber das Department of Psychology gegründet. Seinem Ruf folgten sehr bald der Neuroanatom Walle Nauta, der Experimentalpsychologe Richard Held, der Neurophysiologe Peter Schiller und später Emilio Bizzi, was den Beginn einer experimentell ausgerichteten Ära der Psychologie markierte. Außerdem organisierte Teuber auf dem MIT-Campus ein klinisches Forschungszentrum für Patienten mit Hirntrauma. Natürlich hat es mich auch begeistert, wenn ich vormittags ein neues Buch im Buchladen des MIT in die Hand nahm, und abends den Autor bei einem get-together persönlich treffen konnte! Teuber lud seine Studenten,

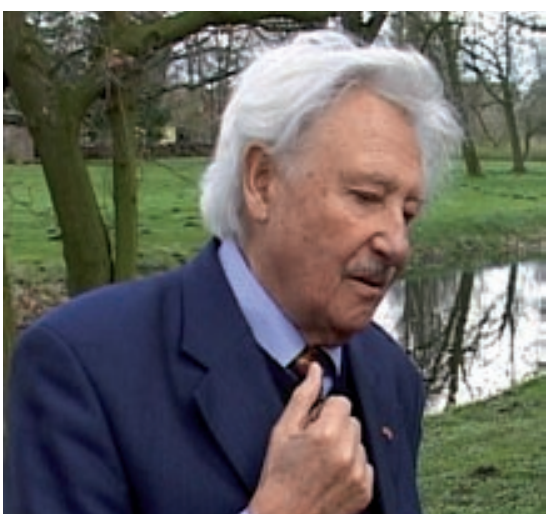

Prof. Georg W. Kreutzberg ist ein weltweit anerkannter Experte auf dem Gebiet von Degeneration und Regeneration im Zentralnervensystem. Er gehört zu den von ISI identifizierten hundert „Highly Cited Researchers" auf dem Gebiet der Neurowissenschaften. Von 1999-2000 war Georg Kreutzberg Präsident der Neurowissenschaftlichen Gesellschaft.

Mitarbeiter und Gäste wöchentlich zu sich nach Hause ein, wobei man auch die ebenso breite, wie profunde Bildung des aus Nazideutschland emigrierten Teuber bewundern und genießen konnte.

Neuroforum: In welchem Methodenspektrum waren Ihre eigenen Arbeiten am MIT angesiedelt?

Georg Kreutzberg: Ich hatte next door zu Walle Nauta's Arbeitsgruppe das große Glück, mit Joe und Betsy Altman Experten für autoradiographische Methoden zu treffen. Zunächst interessierte ich mich vor allem für die Möglichkeit, mit der radioaktiven Thymidin-Markierung Zellteilungsaktivität im Gehirn nachzuweisen.

Neuroforum: Sie haben die Ergebnisse 1966, nach Ihrer Rückkehr ans Max-Planck-Institut für Psychiatrie in München in der Acta Neuropathologica publiziert und damit als, single author" eine wirklich brisante Entdeckung beschrieben, nämlich die Proliferation von Gliazellen im Fazialiskern der Ratte nach Axotomie. Diesen als „Facialis-Modell“ in die Literatur eingegangenen Ansatz bei der Erforschung zentralnervöser Degeneration und Regeneration hatten Sie schon vor Ihrer Bostoner Zeit, als Assistent in der Arbeitsgruppe des Neuropathologen Gert Peters am Max-Planck-Institut für Psychiatrie in München entwickelt. Man kann wohl tatsächlich sagen: Sie kamen nach Boston mit einer Idee und erhielten dort schnellen Zugang zu den besten Methoden, und dies ohne den heute ganz sicher notwendigen „Tribut“" in Form 


\section{Spektrum Sachbücher}

\section{Bildungsfutter und Lesespaß in einem:}

\section{Spektrum}

$\mathrm{S}$ a c h b u c h

\section{Von den Knochen bis zur Seele}

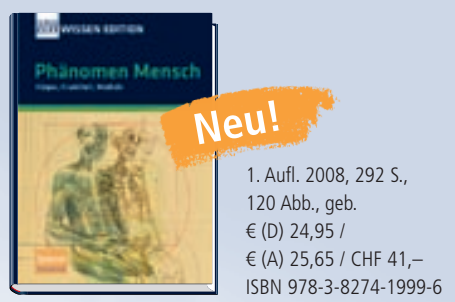

Andreas Sentker / Frank Wigger (Hrsg.)

Phänomen Mensch - Körper, Krankheit, Medizin

Der menschliche Körper ist ein Wunderwerk - und störanfällig. Wie aus einer befruchteten Eizelle ein kompletter Organismus mit Milliarden unterschiedlich spezialisierter Zellen und Tausenden ineinandergreifender Regelkreise wird, ist noch lange nicht verstanden - und die Frage, wie dieses wohlgeordnete System bei Krankheiten durcheinandergerät, ist Gegenstand intensiver Forschung. Phänomen Mensch, der 3. Band der ZEIT WISSEN Edition, zeigt, was Anatomen und Physiologen, Biowissenschaftler und Molekularmediziner heute über den Menschen wissen.

\section{Sind Sie noch derselbe wie vor} einer Minute ... ?

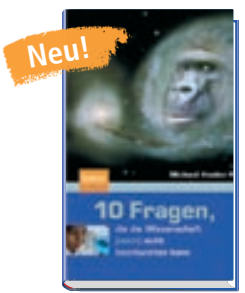

1. Aufl. 2008, 208 S., geb. m. SU $€$ (D) $19,95 / €$ (A) $20,50 /$ CHF 32,50 ISBN 978-3-8274-1979-8

Michael Hanlon

10 Fragen, die die Wissenschaft (noch) nicht beantworten kann

Woraus besteht das Universum? Wie wirklich ist die Realität? Haben Gorillas Humor? Dieses schillernde Buch präsentiert zehn ganz unterschiedliche wissenschaftliche Rätsel, für die die Forschung noch keine Lösung gefunden hat oder deren Klärung immer wieder "in den nächsten zehn Jahren" erwartet wird. Erfahren Sie in diesem mit einem Augenzwinkern geschriebenen Werk, warum es bisher nicht gelungen ist, jene Wissenslücken zu schließen, und wie die Wissenschaftler an die teils großen, weltbewegenden, teils kleinen, alltagsnahen Fragen herangehen.
Werden wir bald unsterblich sein?

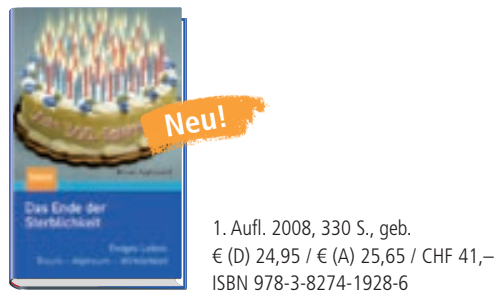

Bryan Appleyard

Das Ende der Sterblichkeit

Das Streben nach Unsterblichkeit, nach ewiger Jugend zieht sich durch die gesamte Menschheitsgeschichte. Aber erst in den letzten Jahrzehnten haben die medizinischen Wissenschaften so rasante Fortschritte gemacht, dass heute manch einer der Ansicht ist, der erste Mensch, der 1000 Jahre alt werde, sei bereits geboren. „Das Ende der Sterblichkeit" zeichnet nicht nur die facettenreiche Geschichte unserer Unsterblichkeitsobsession nach, sondern liefert auch eine spannende Momentaufnahme der Versuche, diesen Traum zu verwirklichen.

\section{Krankheitserreger: nicht Feinde,} sondern Partner des Menschen?

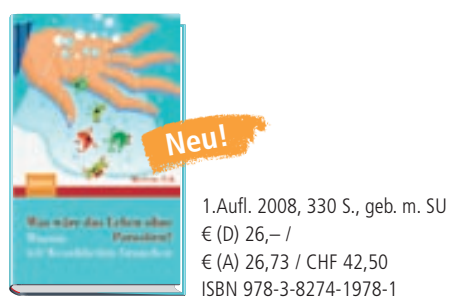

Marlene Zuk

Was wäre das Leben ohne Parasiten?

Wir empfinden Krankheit als unseren Feind, Keime und Infektionen als etwas, das es zu bekämpfen gilt. Aber tun wir ihnen damit vielleicht Unrecht? Mit ihrem unterhaltsamen und fesselnden Buch bringt uns die Evolutionsbiologin Marlene Zuk dazu, unsere instinktiven Gefühle neu zu überdenken. Für sie ist Krankheit unser Partner, nicht unser Feind. Anhand neuester Forschungsergebnisse und ungewöhnlicher Studien beschreibt die Autorin die Bedeutung von Krankheit. Das Buch bringt letztendlich unser Bild vom „bösen Parasiten" gehörig ins Wanken.

\section{Warum gute Menschen böse werden ...}

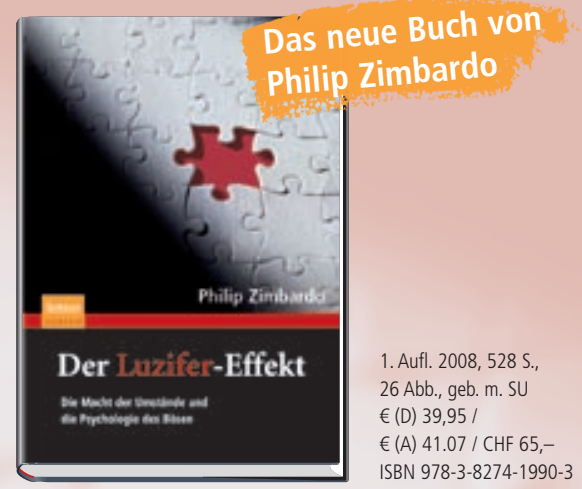

Philip Zimbardo

Der Luzifer-Effekt

Was bringt gute Menschen dazu, Böses zu tun? Wie können normale Menschen dazu verleitet werden, unmoralisch zu handeln? Wo liegt die Grenze zwischen Gut und Böse, und wer läuft Gefahr, sie zu überschreiten?

Der renommierte Sozialpsychologe Philip Zimbardo erläutert in seinem neuen Buch Der Luzifer-Effekt, wie wir alle für die Versuchungen „der finsteren Seite" anfällig sind - und die unzähligen Gründe dafür. Anhand historischer Beispiele sowie seiner eigenen bahnbrechenden Forschungen führt er detailliert aus, wie situative Kräfte und gruppendynamische Prozesse zusammenwirken können, um aus anständigen Männern und Frauen Ungeheuer werden zu lassen.

Der Luzifer-Effekt ist eine schockierende

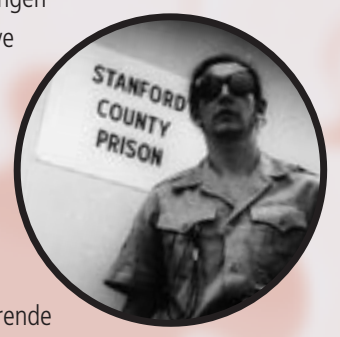
und fesselnde Studie, die ein neues Licht auf unsere Sicht des menschlichen Verhaltens wirft.

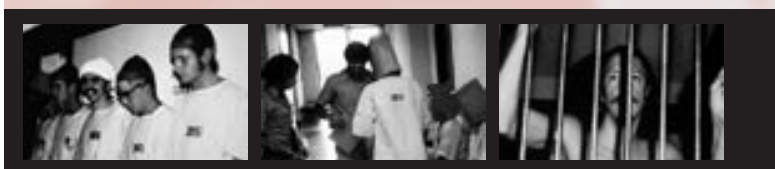

Das Stanford Prison Experiment: eine Studie, in der eine Gruppe freiwilliger Studenten zufällig in "Wärter" und "Häftlinge" aufgeteilt wurde, um dann in einem simulierten Gefängnis zu arbeiten und zu leben. Das auf zwei Wochen angelegte Experiment musste nach 6 Tagen abgebrochen werden, da normale Studenten sich in brutale, sadistische Wärter oder emotional gebrochene Gefangene verwandelt hatten. 
von multiplen Ko-Autorenschaften zu zahlen. Hat der „Bostoner Stil“, Wissenschaft zu betreiben, ihre eigene Aufbauarbeit als MaxPlanck-Direktor in München beeinflusst, und wenn ja, worin bestand dieser Einfluss?

Georg Kreutzberg: Ganz sicher war dieser Einfluss enorm. Worin hat er bestanden? Also, ich glaube, ich habe in Boston vor allem gelernt, WIE man wissenschaftlich arbeitet, wie die Umsetzung einer faszinierenden Idee in eine technisch machbare Studie in einer vertretbaren Zeit gelingen kann. Diese Erfahrungen wurden noch vertieft, denn es folgte $(1967 / 68)$ ein weiterer USA-Aufenthalt, diesmal auf Einladung von Paul Weiss, der mich als guest investigator an die RockefellerUniversität nach New York brachte.

Neuroforum: Ihr „Fazialis-Modell“ hatte ja Hinweise geliefert, dass im Inneren des Axons Material zum Muskel transportiert wird, und Sie hatten mit Hilfe des am Tubulin angreifenden Zytostatikums Colchizin Hinweise erhalten, dass die Mikrotubuli am axonalen Transport beteiligt sind. Diese Arbeit wurde 1969 in PNAS publiziert. Auch hier sind sie alleiniger Autor. - Was nun würden Sie als die entscheidende Mitgift Ihrer New Yorker Zeit ansehen?

Georg Kreutzberg: Vor allem verlor ich jegliche Scheu vor dem Einsatz immer wieder neuer Methoden, auch solcher, die einem Morphologen nicht gerade in die Wiege gelegt werden. Ich glaube, die besonders erfolgreichen Einrichtungen der neurowissenschaftlichen Forschung sind alle interdisziplinär und damit in besonderem Maße auf die gute Kommunikation und Koordination aller an einem Projekt beteiligten Wissenschaftler angewiesen. Dabei muss der Transfer von wissenschaftlichem know how nicht zwangsläufig zu Mitautorenschaft führen. Es ist letztlich eine Frage der wissenschaftlichen Ehre, als Autor nur auf Publikationen zu sein, $\mathrm{zu}$ denen ein aktiver inhaltlicher Beitrag geleistet wurde.

Neuroforum: Glauben Sie, dass sich die Ethik des wissenschaftlichen Arbeitens in den letzten 40 Jahren gewandelt hat?

Georg Kreutzberg: Auch Ethik wandelt sich und steht in Wechselwirkung mit dem Zeitgeist. Damals blieb vieles doch der Intuition, dem individuellen Wertekompass, d.h. dem Empfinden des jeweiligen Wissenschaftlers überlassen. Der zum Teil ganz gewaltige Druck, den der Wettbewerb um Fördermittel mit sich bringt, die Erfahrung, dass der Einsatz von Hochtechnologien, wie auch das individuelle Fortkommen von Publikationsleistungen abhängig sind, hat uns allerdings in einigen Fällen die Fragilität unseres Systems der Kontrolle von individuellem Tun im Dienste der Wissenschaft vor Augen geführt.

Neuroforum: Sie haben sich in diesem Bereich ja besonders engagiert...

Georg Kreutzberg: Ja, ich fand es notwendig und wichtig, mich im Auftrag der Max-Planck-Gesellschaft und anderer wissenschaftlicher Institutionen an der Ausarbeitung einer allgemein verbindlichen Richtlinie zur Sicherung guter wissenschaftlicher Praxis zu beteiligen. Zuvor war ich involviert in die Sanktionierung eines schweren Betrugsfalls, der im Wesentlichen eine ausländische Universität betraf. Hier haben wir dem Verursacher klargemacht: $»$ Never ever! «. Aber natürlich geht es vor allem um die Prävention von Verfälschungen wissenschaftlicher Ergebnisse durch Plagiat, Erfindung oder Schönung von Daten. Die Kontrolle von Primärdaten durch kurze Wege von der bench zum office, von der Produktion zur Präsentation der Ergebnisse ist hier von großer Bedeutung!

Neuroforum: Lassen Sie uns noch einmal auf Ihre eigene Forschungstätigkeit zurückkommen. Mit welcher Leistung wurde Ihnen die größte Anerkennung zuteil, und in welchem Beitrag steckt das meiste „Herzblut"?

Georg Kreutzberg: Sie haben recht, nicht immer deckt sich die Akzeptanz einer Arbeit mit der subjektiven Wahrnehmung des geleisteten Jobs! Wenn wir uns der vielleicht nicht ganz harmlosen Werkzeuge zur Beurteilung des wissenschaftlichen Impacts einer Publikation bedienen wollen, dann wäre das in meinem Falle ein Artikel in TINS (1996) mit dem Titel „Microglia: a sensor for pathological events in the CNS". Dieser invited review ist mir insofern wichtig, als er etwa 30 Jahre Forschung meiner Arbeitsgruppe am MPI für Psychiatrie in München reflektiert, und das schnell fortschreitende Verständnis bei einem Problem verdeutlicht, das ich bereits in jungen Jahren als MEIN PROBLEM identifizieren konnte: die Reaktion von zentralnervösem Gewebe auf Schädigung. Mit den frühen Thymidin-Studien hatten wir ja bald herausgefunden, dass es sich bei den proliferierenden Zellen vor allem um Mikroglia handelt. Das charakteristischste Merkmal der Mikroglia ist, dass sie bei Einwirkung von Noxen jedweder Art von einem scheinbar ruhenden in einen aktivierten Zustand übergeht. Letzterer ist gekennzeichnet durch die
Aufregulation einer Reihe von Molekülen, die wir und andere zunächst mit immunhistochemischen, dann mit den Methoden der Molekularbiologie identifizieren konnten. Wir haben zum Beispiel erkannt, dass Mikrogliazellen nach Aktivation das amyloid precursor protein (APP) de novo produzieren, was bei neurodegenerativen Erkrankungen, wie der Alzheimer-Erkrankung, eine wichtige Rolle spielt.

Neuroforum: Sie gelten auch als Experte für Elektronenmikroskopie und haben am MPI für Psychiatrie die Abteilung Neuromorphologie geleitet. Was hat denn der Blick des Neuroanatomen Kreutzberg auf die U1trastruktur des Facialis-Kerns im Hirnstamm von Kaninchen, Ratte und Maus gebracht?

Georg Kreutzberg: In der Tat, da haben wir entgegen den üblichen Prinzipien der visuellen Wahrnehmung einiges gesehen! Es ist ja so, dass einem viel schneller auffällt, wenn etwas hinzukommt, das normalerweise nicht ins Bild gehört. Nach der Axotomie ist aber etwas verschwunden, nämlich die Synapsen - und dies war vielleicht der Grund, warum vor uns kaum jemand auf ein Phänomen aufmerksam wurde, das wir und andere später als „synaptic stripping“ bezeichnet haben: der Rückzug vesikelgefüllter präsynaptischer Terminalen vom Zellkörper und den Stammdendriten axotomierter Motoneurone. Dieses synaptic stripping konnte durch Blockade der Mikrogliaaktivierung, z.B. durch Adriamyzin, verhindert werden. Es ist offensichtlich, dass dies eine notwendige Voraussetzung für die synaptische Transmitterfreisetzung und damit die Aktivität der Fazialis-Motoneurone ist, welche ihrerseits die Re-Innervation des nach Läsion denervierten Muskels stark beeinflusst. Ausserdem erklärte der Synapsenschwund auch die klinischen Symptome der Dyskinesien.

Neuroforum: Wie ist es dann mit dem neuronalen Transport weitergegangen? Wir haben hier ein Buch aus dem Jahr 1975 ausgegraben, edited by Georg W. Kreutzberg, und erschienen by Raven Press, New York. Der Titel ist „Physiology and Pathology of Dendrites". Da erscheinen Sie zusammen mit Hans-Dieter Lux und Peter Schubert in mehreren Kapiteln zum Thema „Dendritischer Transport".

Georg Kreutzberg: Ja, es zeigte sich sehr bald, dass Tritium-markierte Aminosäuren oder Nukleotide sehr gut geeignet sind, neben der Geschwindigkeit intrazellulärer Transportvorgänge auch die Transportwege $\mathrm{zu}$ bestimmen. Uns interessierten in dieser 
Phase die intradendritischen Transportvorgänge, da diese für die Stabilisierung aktiver Synapsen sehr wahrscheinlich von großer Bedeutung sind. Die an der Studie beteiligten Neurophysiologen, vor allem Hans Dieter Lux und Peter Schubert, hatten mit der Mikroelektrodentechnik ein neues Werkzeug, 3H-Adenosin oder -Leuzin in die Zellen hineinzubringen. Das lieferte mir die Möglichkeit, Serienschnitte dieser Neurone in einem aufwendigen Verfahren so zu rekonstruieren, dass sich der gesamte Dendritenbaum darstellte. Damit ergab sich erstmals die Möglichkeit, die geometrischen Längen mit den vorher bestimmten elektrotonischen Längen direkt in Beziehung zu setzen. Die durch Experimentaldaten abgesicherte mathematische Kabelanalyse der dendritischen Transfereigenschaften lieferte dann auch eine Antwort auf die Frage, welchen Einfluss die Position einer Synapse auf den langen und vielfach verzweigten Ästen eines Dendritenbaums hat.

Neuroforum: Herr Kreutzberg, Sie gehören eindeutig zu den besonders glücklichen Forschern, die ihr Thema in jungen Jahren gefunden haben und nach optimaler Auslanderfahrung zum GLEICHEN THEMA auch am GLEICHEN ORT geforscht haben. Eine solche Vita ist natürlich zu jeder Zeit eine Ausnahmeerscheinung, trotzdem mag der eine oder andere Ihrer heute tätigen und nicht selten etwas respektlosen „Enkel“ auch eine gewaltige Begünstigung durch die historischen Umstände erkennen. Würden Sie dem beipflichten?

Georg Kreutzberg: Ein bisschen Fortune kann im Leben ja nicht schaden! Aber der von Neugier und Forscherdrang motivierte Wissenschaftler wird zu allen Zeiten so viel arbeiten, wie er sich physisch zumuten kann. Er bedarf auch nicht des existentiellen Druckes als Antrieb für sein alltägliches
Handeln, im Gegenteil! Wahr ist andererseits, dass die moderne neurobiologische Grundlagenforschung weit stärker eines professionellen Managements bedarf, als das noch vor 30 Jahren der Fall war. In kurzen Zyklen müssen immer wieder neue Experten unterschiedlichsten Kalibers, auch verschiedener Nationalitäten und Wertesysteme für die jeweilige Aufgabe gefunden werden, was dem Ausleben von Individualität heute vielleicht engere Grenzen setzt. Trotzdem ist und bleibt jede wirklich kreative Leistung immer ein Produkt des freien Denkens, was mitunter sogar die Entthronung früherer Helden bedeuten kann.

Neuroforum: Wir nehmen an, die meisten deutschen Lehrstühle für Neuroanatomie oder Neuropathologie sind inzwischen mit ihren Schülern besetzt?

Georg Kreutzberg: Da berühren Sie einen für mich doch einigermaßen schmerzhaften Punkt! Ich darf vielleicht 20 Wissenschaftler als ,Schüler" bezeichnen, weil sie als Doktoranden, Postdocs oder Gastwissenschaftler über eine längere Zeit im Labor waren. Der weitere Werdegang meiner ehemaligen Mitstreiter war mir immer wichtig, deswegen kann es mich auch nicht freuen, dass mit zwei Ausnahmen keiner von ihnen heute einen Lehrstuhl an einer deutschen Universität bekleidet. Die meisten von ihnen sind im Ausland tätig, in die klinischen Fächer oder die Industrie abgewandert.

Neuroforum: Wollen Sie uns vielleicht einige ihrer wichtigsten Schüler und Wegbegleiter nennen?

Georg Kreutzberg: Mit den Namen ist das so eine Sache, ich möchte vorsorglich anmerken, dass die folgende Liste nicht vollständig sein kann, aber sicher gehören dazu auch R. Banati, M. Graeber, C.A. Haas, K.S. Lee,
W. Nacimiento, J. Priller, G. Raivich, M. Reddington, S. Schön, P. Schubert, W.J. Streit, W. Tetzlaff und D.G. Weiss.

Neuroforum: Wer war denn ihr erster Doktorand?

Georg Kreutzberg: Mein erster Doktorand war eine ,sie“, und sie ist immer noch an meiner Seite, als meine Ehefrau und als Mutter meiner beiden wunderbaren Söhne. Sie hat die aktive Forschungstätigkeit schon lange aufgegeben, aber nicht ihre Neugier und Energie. Sie arbeitet immer noch als freischaffende Medizinjournalistin. Sie fährt auch inzwischen besser Ski als ich, was ich aber leicht verschmerzen kann.

Neuroforum: Herr Kreutzberg, wir danken Ihnen sehr für dieses Gespräch!

Das Gespräch führte Rosemarie Grantyn. RG ist Professor emeritus am Institut für Neurophysiologie der Charité Berlin. Sie forscht auf dem Gebiet der Entwicklungsphysiologie, mit dem Schwerpunkt GABAerge Hemmung.

\section{Kurzbiografie}

Georg Kreutzberg wurde 1932 in Ahrweiler geboren, promovierte 1961 in Freiburg/Br. zum Dr. med. und habilitierte sich 1971 in Neuropathologie an der TU München. 1978 wurde er Wissenschaftliches Mitglied des Max-Planck-Instituts für Psychiatrie, wo er bis zu seiner Emeritierung (2000) als Direktor der Abteilung für Neuromorphologie tätig war. Von 1985 bis 1995 wirkte er außerdem als geschäftsführender Direktor des Theoretischen Instituts des MPI für Psychiatrie/ Neurobiologie in München-Martinsried. 2007 erhielt er das Verdienstkreuz 1. Klasse des Verdienstordens der Bundesrepublik Deutschland, womit auch seine umfangreiche ehrenamtliche Tätigkeit gewürdigt wurde.

\section{Stipendien für die Göttinger Jahrestagung 2009}

Bewerbungsschluss ist der 15. Oktober 2008.

Bitte senden Sie Ihre Bewerbung per E-Mail an:

Neurowissenschaftliche Gesellschaft e.V. Max-Delbrück-Centrum für Molekular

Meino Alexandra Gibson

Die Neurowissenschaftliche Gesellschaft e.V. stellt wieder Reisestipendien für die Teilnahme am 8th Göttingen Meeting of the German Neuroscience Society (25. - 29. März 2009) zur Verfügung.

Bewerben können sich

- Doktoranden und Postdocs,

- die max. 35 Jahre alt sind.

Das Reisestipendium in Höhe von 300 Euro wird in bar auf der Tagung ausgezahlt. Die Bewerbung sollte folgende Unterlagen enthalten:

- einseitiger Lebenslauf

- Publikationsliste

- Kopie des Abstracts

- ein kurzes Empfehlungsschreiben
Robert-Rössle-Str. 10, D-13092 Berlin

E-Mail:gibson@mdc-berlin.de

Postalisch eingesandte Bewerbungsunterlagen können nicht bearbeitet werden, nur per E-Mail eingesandte Bewerbungen kommen in den Auswahlprozess. 